\title{
Optical and photoelectrical properties of lamellar gallium sulfide single crystals irradiated by $\gamma$-quanta
}

\author{
R.S. Madatov, B.G. Tagiyev, A.I. Najafov, T.B. Tagiyev, I.A. Gabulov, Sh.P. Shakili \\ Institute of Radiation Problems of Azerbaijan National Academy of Sciences \\ 9, F. Agayev str., Baku, AZ 1143, tel./fax: (99412) 4398318
}

\begin{abstract}
The influence of $\gamma$-quanta irradiation on photoelectrical and optical properties of lamellar GaS single crystals at different temperatures has been investigated. It is determined that the irradiation of pure crystals at the radiation dose equal to $30 \mathrm{krad}$ results in the creation of shallow compensative acceptors, which are photoactive recombination centers ( $r$-centers), and as a result of this both the photosensitivity and a luminescence connected with $r$-centers are increased. Irradiation with a radiation dose more than $100 \mathrm{krad}$ results in the quenching of both photosensitivity and recombination luminescence due to formation of complexes $\left[\mathrm{V}_{\mathrm{Ga}} \mathrm{V}_{\mathrm{S}}\right]$. It is proposed that radiative recombination centers arising in the course of irradiation is conditioned by sulfur hole and interstitial gallium atoms.
\end{abstract}

Keywords: photoluminescence, photoconductivity, single crystal, exciton, intra-central transition.

Manuscript received 27.01.06; accepted for publication 29.03.06.

\section{Introduction}

In accordance with [1-3] $\mathrm{A}^{\mathrm{III}} \mathrm{B}^{\mathrm{IV}}$ compounds are interested as promising materials to create semiconductor detectors of elementary particles and hard electromagnetic radiation. Increased interest to these compounds is caused by circumstance that though their strong defectiveness they have high photosensitivity in visible, infrared, roentgen and gamma-ray spectral ranges [3-9]. These preliminary data pointed at the possible prospective using the lamellar semiconductor compounds for the development of photoelectrical devices, radiation sources and radiation detectors. In this connection, the research of their photoelectrical properties behavior at ionizing radiation is actual.

The research results of optical and photoelectrical characteristics of lamellar GaS single crystals are irradiated by gamma-quanta with the purpose of local levels detection in the crystal forbidden-zone are given in this paper.

Investigated $p$-GaS single crystals were grown using the Bridgman method at the Institute of Radiation Problems of Azerbaijan National Academy of Sciences. Sulfur surplus $(1.5 \%)$ is used during growth of single crystals with the purpose to determine the hole filling possibility by sulfur atoms. It was experimentally determined that effective filling of holes occur at annealing temperatures 500 to $700{ }^{\circ} \mathrm{C}$. Specific resistances of the samples along and perpendicularly to $c$ axis at the room temperature are $2 \cdot 10$ and $3 \cdot 10^{7} \mathrm{Ohm} \cdot \mathrm{cm}$, respectively. Indium was used as a material for ohmic contacts. Indium was fused into $\mathrm{GaS}$ surface at $150^{\circ} \mathrm{C}$. Irradiation of the samples by gammaquanta with energy $1.3 \mathrm{MeV}$ was carried out using $\mathrm{Co}^{60}$ at $300 \mathrm{~K}$. The crystals were cooled by liquid nitrogen vapor during irradiation and, as a result, the temperature of crystals was not higher than $290 \mathrm{~K}$.

\section{Experimental results}

The investigations of photoconductivity and photoluminescence in the range of wavelengths $0.4 \ldots 1.0 \mu \mathrm{m}$ at the temperatures 120 and $300 \mathrm{~K}$ have been carried out to reveal local levels in the obtained GaS single crystals.

Spectral dependences of photoconductivity for obtained GaS single crystals are presented in Fig. 1. It is necessary to note that initial GaS samples have photoconductivity maxima near the fundamental absorption edge at $\lambda=0.51 \mu \mathrm{m}$. In addition, observed are intensive impurity peaks with maxima at $\lambda=0.61 \mu \mathrm{m}$ and $\lambda=0.70 \mu \mathrm{m}$ (Fig. 1, curve 1). These maxima correspond to the optical transition from the acceptor level to the conduction band. Activation energy of the 


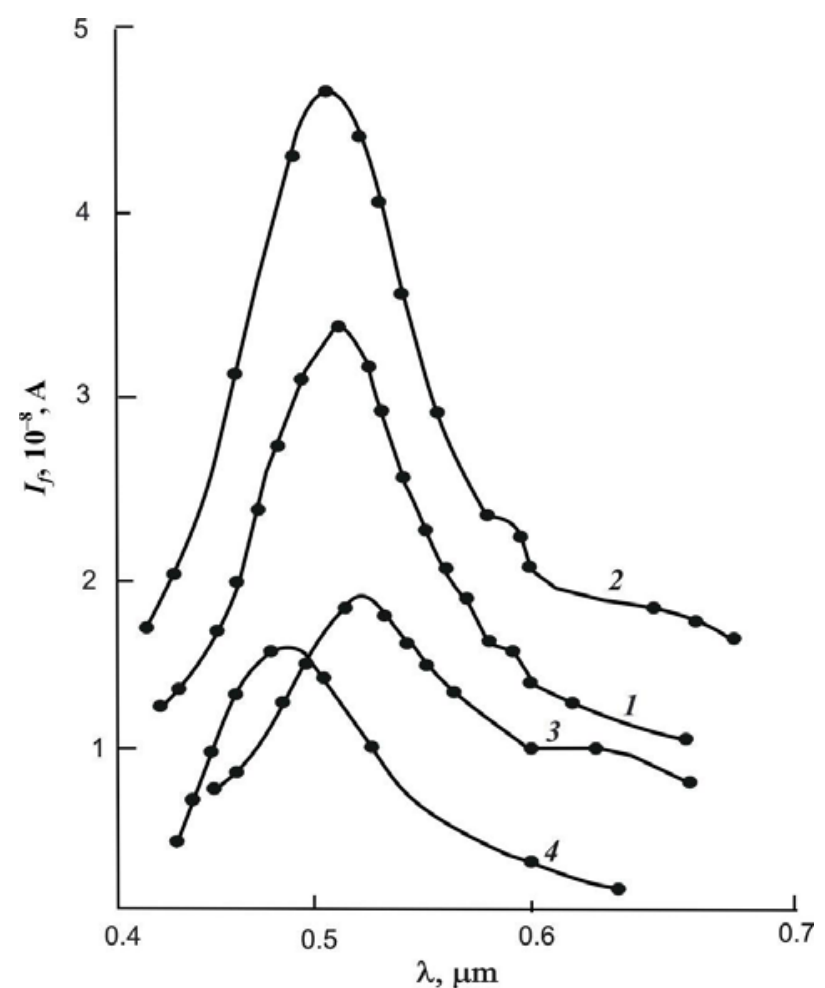

Fig. 1. Spectral distribution of photoconductivity for $\mathrm{GaS}$ single crystals: 1,2 - without irradiation; 3, 4 - irradiated by gamma-quanta (30 krad); 1,3 at $300 \mathrm{~K} ; 2,4$ at $120 \mathrm{~K}$.

levels is equal to 0.50 and $0.74 \mathrm{eV}$, respectively. It coincides with the values given in $[3,4]$. Photocurrent increases approximately by $30-40 \%$ after irradiation of the samples by gamma-quanta with the dose equal to $30 \mathrm{krad}$. In this case, the maximum intensity at $0.61 \mu \mathrm{m}$ decreases and with increasing irradiation dose gradually decrease and disappear at the dose equal to $100 \mathrm{krad}$ (Fig. 1, curves 2-4). The peak in the vicinity of $0.74 \mathrm{eV}$ is displaced to the short waves and appeared at $\lambda=0.82 \mu \mathrm{m}$. It is seen from Fig. 1 that further irradiation decreases the photoconductivity of $\mathrm{GaS}$ in all the investigated spectral region (curves 3,4 ). It is indicative of generation of a high concentration of recombination centers with the large capture cross-section for electrons.

The temperature dependences of photocurrent in the initial and irradiated GaS samples at $\lambda=0.51 \mu \mathrm{m}$ are given in Fig. 2. The samples are irradiated by gammaquanta with the doses equal to 30 and $100 \mathrm{krad}$. As it is seen, the irradiation does not influence on the temperature dependence of photocurrent in $\mathrm{GaS}$ samples. It is observed temperature quenching of photocurrent at the temperature higher than $170 \mathrm{~K}$. Such sensitivity changes connected mainly with rearrangement of intrinsic defect levels in the forbidden zone and changing in hole filling ratio of sensitivity centers in GaS. Photoluminescence spectra of the investigated samples at $77 \mathrm{~K}$ are given in Fig. 3. Heliumcadmium laser $(\lambda=0.3716 \mu \mathrm{m})$ was used for excitation. Intensive exciton emission bands with $\lambda_{1}=0.48 \mu \mathrm{m}$ are

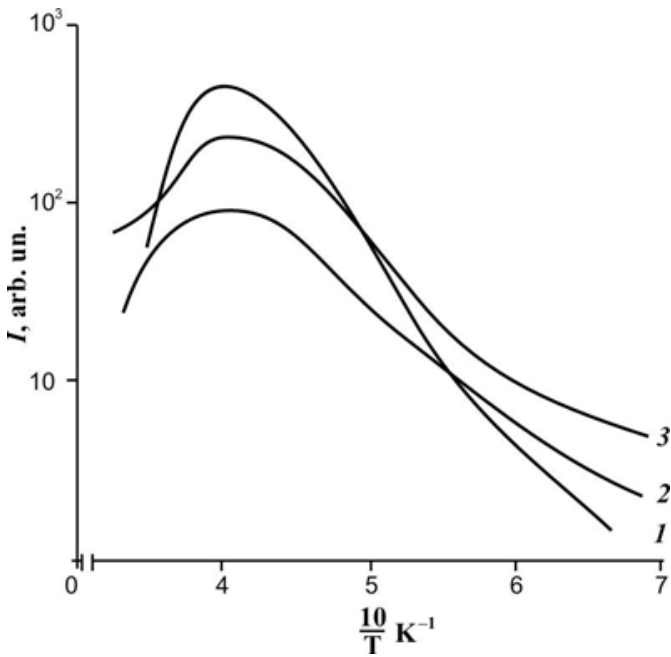

Fig. 2. Temperature dependences of photocurrent $\left(\lambda_{\max }=\right.$ $=0.51 \mu \mathrm{m}$ ) for single crystals GaS: 1 - before irradiation; 2 $30 \mathrm{krad} ; 3-100 \mathrm{krad}$.

observed in both $\mathrm{GaS}$ crystals irradiated by low doses and unirradiated samples. The wide structureless band having peaks of the considerable intensity located at $\lambda_{1}=0.48 \mu \mathrm{m}, \lambda_{2}=0.52 \mu \mathrm{m}$ and $\lambda_{3}=0.66 \mu \mathrm{m}$ raised after irradiation of samples with $30 \mathrm{krad}$ dose. The observed peak $\lambda_{3}=0.66 \mu \mathrm{m}$ disappears at high irradiation doses (curve 3,100 krad), and the dependence behavior gets its initial look similar to that before irradiation. The dependence of the irradiation intensity and photosensitivity on the irradiation dose is shown in Fig. 4. It is seen that at low irradiation doses up to $30 \mathrm{krad}$, it is observed heightened intensity band, and further increasing the irradiation dose results in the decreased intensity. The photoconductivity dependence of irradiated samples demonstrates the same behavior.

\section{Discussion of the obtained results}

The researches of stationary characteristics of photoconductivity and photoluminescence allow to determine a recombination model in $\mathrm{GaS}$ single crystals including post-gamma-irradiation influence. The observation of such phenomena as radiation and photoluminescence of the crystals as well as thermal quenchering of photocurrent can be explained within the framework of three-level recombination diagram containing low $-r$, fast $-s$ and capture levels $-t$ for majority charge carriers. It is known [11] that in the thermodynamical state of equilibrium for implementation of high photoconductivity the levels of $r$ and $s$ should be completely filled by the holes. In this case, the electron concentration $N_{r 0}$ should correspond to the following conditions:

$N_{r 0} \ll<P_{r 0}=N_{r}, N_{s 0}<<N_{r}$,

where $N_{r}$ and $P_{r 0}, N_{s}$ are the concentrations of $r$ and $s$ centers, respectively. Majority charge carriers for GaS are holes, and the condition $N_{a}>N_{d}$ is carried out. Illuminating of the samples leads to the optical 


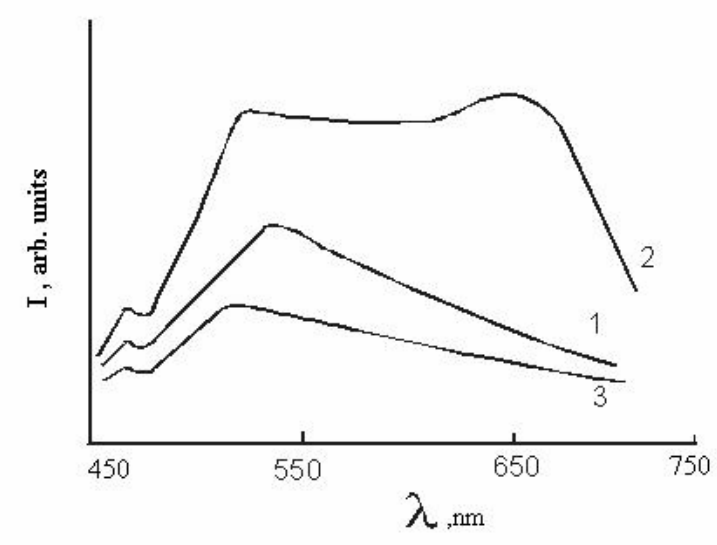

Fig. 3. Photoluminescent spectra of GaS single crystals: 1 before irradiation; $2-30 \mathrm{krad} ; 3-100 \mathrm{krad}$.

recharging of local levels and, as a result, the filling of these levels significantly differs from its dark value.

The complex researches at different temperatures were carried out in order to determine the cause of photoconductivity changing in the irradiated $p$-type GaS samples. It is ascertained that at low irradiation doses (up to $20 \mathrm{krad}$ ) the photosensitivity in the range of intrinsic photoconductivity and the intensity of bands with the peaks located at $\lambda_{f}=0.62 \mu \mathrm{m}$ (Fig. 1, curve 2) and $\lambda_{\mathrm{I}}=0.48 \mu \mathrm{m}$ (Fig. 3, curve 1) are not practically changed, and it is an evidence of low speed photosensitivity of the injected radiation defects. An increase in photosensitivity for $\lambda_{\max }=0.51 \mu \mathrm{m}$ and decrease for $\lambda_{f}=0.62 \mu \mathrm{m}$ occur with increasing gammairradiation dose up to $30 \mathrm{krad}$. This fact is explained by the increase of low recombination centers in the composition where $\mathrm{V}_{\mathrm{S}}$ is included, and the decreasing $\mathrm{V}_{\mathrm{Ga}}$ concentration. On the basis of the obtained results, it is difficult to give a conclusion about the nature of $r$ centers, but it is possible to suppose that complex defects with sulfur and gallium vacancies are responsible for these centers. In fact, the decrease of the impurity peak $(0.62 \mu \mathrm{m})$ testifies to the decrease of the $\mathrm{V}_{\mathrm{Ga}}$ concentration, it seems due to interaction with $\mathrm{Ga}_{i}$ [5] . The researches results of photoluminescent spectra for irradiated GaS crystals (Fig. 3, curves 2, 3) showed the creation of radiation defects. It is seen from Fig. 3 that in the excitation spectrum of luminescence for the irradiated sample $(30 \mathrm{krad})$ additional high intensity maxima with $\lambda_{2}=0.53 \mu \mathrm{m}$ and $\lambda_{3}=0.66 \mu \mathrm{m}$ are formed in addition to the exciton band $\left(\lambda_{1}=0.48 \mu \mathrm{m}\right)$. It is necessary to note that shortwave peak with $\lambda_{1}=0.48 \mu \mathrm{m}$ is conditioned by radiative recombination of free electrons and its energetic position coincides with that for the exciton peak $n=1$ in the absorption spectrum $[2,6]$. It is known [12] that the boundary energy of electrons required for sulfur atoms to be displaced into the interstitial site is two times less than the energy required for gallium atoms to be displaced. Therefore, we can suppose that the acceptor centers (interstitial sulfur atoms $\mathrm{S}_{i}$ ) are responsible for the band at $0.53 \mu \mathrm{m}$.
In this case, radiation occurs at the recombination of free electrons with holes that are captured by the acceptor centers $\mathrm{S}_{i}$. The shift of the luminescence peak $(0.53 \mu \mathrm{m})$ to the shortwave side of the spectrum and the decrease of its intensity with increasing irradiation dose (Fig. 3, curve 3) can be explained by shielding action of charged holes on the radiation centers, which are $\mathrm{Ga}_{i}$ [7] and removal of $\mathrm{S}_{i}$ to different sinks, which could be $\mathrm{V}_{\mathrm{S}}$, defect cluster, dislocations and etc. It is worth to note that the complex with $\mathrm{Ga}_{i}^{+}$atoms is responsible for luminescence band $0.66 \mu \mathrm{m}$. The decrease of the luminescence band $(0.66 \mu \mathrm{m})$ intensity in the irradiated $\mathrm{GaS}$ crystals (100 krad) is connected with complex dissociation, as a result of that $\mathrm{Ga}_{i}^{+}$atoms annihilate and $\mathrm{V}_{\mathrm{Ga}}$ are formed. It is seen from Fig. 2 (curve 1) that TGF is observed in initial crystals at temperatures $T>200 \mathrm{~K}$ due to the development of thermal generation of electrons, which forms $r$-levels inside C-zone and their further capture at $s$-levels. On temperature decreasing below $200 \mathrm{~K}$, the photocurrent decreases, which shows the localization of holes at $t$-levels and corresponding electrons at $r$-recombination levels. As a result of $N_{r}=N_{t}$ formation accordingly to [11], the decrease of both the hole lifetime and photocurrent occurs. It is seen from Fig. 2 (curves 2,3) that the irradiation does not influence on the behavior of the photocurrent temperature dependence, and TGF is observed at temperatures above $240 \mathrm{~K}$. It means that irradiation by gamma-quanta leads to the radiation sensitization in the temperature range above $170 \mathrm{~K}$. Such change of sensitivity is connected with the change of the hole filling degree of sensitivity centers in GaS as well as GaSe and GaTe [10]. Irradiation by gamma-quanta creates shallow capture levels with the ionization energy $0.23 \mathrm{eV}$. These levels compensate deep levels. The parameters of sensitizing $r$ centers of recombination and trapping were determined: the values of the capture cross-section for electron and hole are equal to $\mathrm{S}_{n r}=2 \cdot 10^{-14}$ and $\mathrm{S}_{p r}=5 \cdot 10^{-19} \mathrm{~cm}^{-2}$,

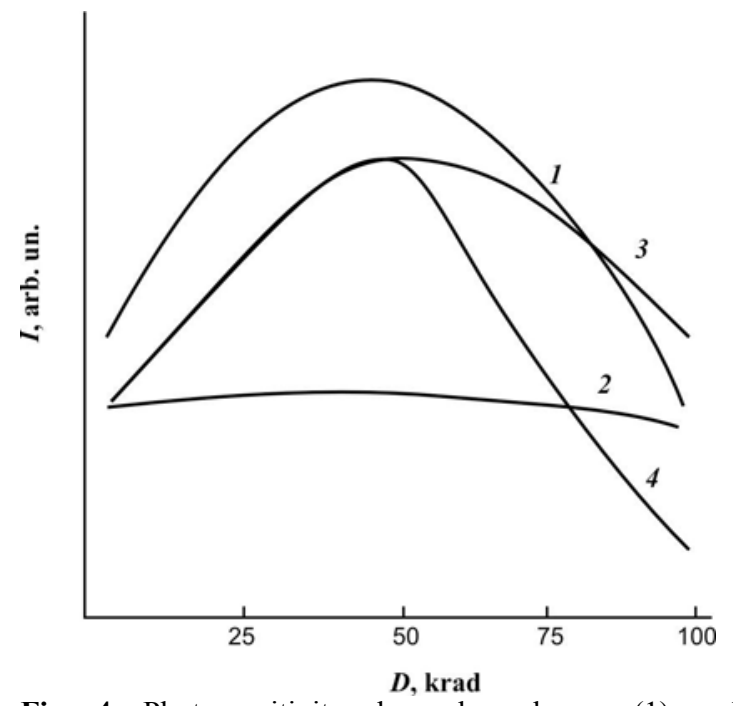

Fig. 4. Photosensitivity dose dependences (1) and the intensity of photoluminescence (2-4) for GaS single crystals : $\lambda=0.48 \mu \mathrm{m}(2) ; 0.53$ (3); 0.66 (4). 
respectively, the concentration of these centers is equal to $2 \cdot 10^{-14} \mathrm{~cm}^{-3}$, and the energy state of trapping levels for holes is $E_{v t}=0.23$ and $0.40 \mathrm{eV}$ and their concentration is $N_{t}=7 \cdot 10^{14}-2 \cdot 10^{15} \mathrm{~cm}^{-3}$. All of these facts show that irradiation by gamma-quanta with low dose leads to the formation of radiative recombination centers in which the band of $0.53 \mu \mathrm{m}$ is determined by donor center with participation of $\mathrm{S}$ vacancy, and the band of $0.66 \mu \mathrm{m}$ is determined by interstitial $\mathrm{Ga}$ atoms. The irradiation by high doses (above $100 \mathrm{krad}$ ) leads to the photosensitivity quenching and recombination luminescence recombination due to the formation of bivacancies $\left[\mathrm{V}_{\mathrm{Ga}}, \mathrm{V}_{\mathrm{S}}\right]$.

\section{Conclusion}

Thus, the irradiation by gamma-quanta of pure crystals leads to the formation of shallow acceptor capture levels with the energy $0.23 \mathrm{eV}$. These levels compensate deep donors, which are sensitizing recombination centers $(r$ centers). It leads to the increasing of photosensitivity and strengthening luminescence that is connected with $r$ centers. Obtained experimental results in the irradiated GaS crystals are explained satisfactorily within the existing model [11].

\section{References}

1. G.A. Akhundov, Ph. D. dissertation, Baku, 1967.

2. V.P. Mushinskiy, M.I. Karaman, Optical properties of chalcogenide gallium and indium. Kishinev, 1973, p. 71.
3. O.Z. Alekperov, M.Z. Zarbaliyev // Izvestiya $A N$ SSSR, Neorganich. materialy 34, No 10, p. 11631167 (1998) (in Russian).

4. G. Fischer, Speculation of the band structure of the layer compounds GaS and GaSe // Helv. Phys. Soc. Acta 36, No 3, p. 1313-1325 (1963).

5. O.Z.Alekperov, M.Z. Zarbaliyev // Izvestiya $A N$ SSSR, Neorganich. materialy 35, No 11, p. 13151320 (1999) (in Russian).

6. H. Kamimara, K. Nakao, Band structure and optical properties of semiconductioning layer compounds GaS and GaSe // J. Phys. Soc. Jpn 24, No 6, p. 1313-1325 (1968).

7. G.B. Abdullayev, A.Z. Abbasova et al. // Fizika tekhnika poluprovodnikov 15, No 6, p 1320-1325 (1981) (in Russian).

8. R.S. Madatov, T.B. Tagiyev, I.A. Kabulov, T.M. Abbasova // Semiconductor Physics, Quantum Electronics and Optoelectronics 6(3), p. 278-281 (2003).

9. T.B. Tagiyev, R.S. Madatov, T.M. Abbasova // Ibid. 5(3), p. 261-263 (2002).

10. Yu.P. Gnatenko, Z.D. Kovalyuk, P.A. Skubenko // Ukr. Fiz. Zhurn. 27(6), p. 838-842 (1982) (in Russian).

11. V.E. Lashkarev, A.V. Lyubcjenko, M.K. Sheykman, Non-equilibrium processes in photoconductors. Naukova Dumka, Kiev, 1981, p. 264 (in Russian).

12. V.V. Emtsev, T.B. Mashovets, Impurities and hole defects in semiconductors. Radio i svyaz', Moscow, 1981, p. 248 (in Russian). 